\title{
Una evaluación del Método Abierto de Coordinación de las políticas de los Estados miembros de la Unión Europea
}

\author{
Jacques Toulemonde \\ Université de Lyon \\ jacques.toulemonde@univ-lyon2.fr \\ Centre for European Evaluation \\ toulemonde@eureval.fr
}

Resumen

Este trabajo tiene su origen en una evaluación llevada a cabo por el autor y varios de sus colegas (desde Euréval y Ramboll Management) para la Comisión Europea, en el año 2007. La evaluación fue aplicada a la coordinación de políticas nacionales, en las áreas de crecimiento económico y empleo. La principal conclusión del informe (<http://ec.europa.eu/economy_finance/>) reside en que el método abierto de coordinación es efectivo al promover el aprendizaje mutuo, el amplio consenso entre las partes interesadas, el reforzamiento de la legitimidad de quienes promueven reformas y, finalmente, el impulso de reformas en las agendas políticas. El instrumento ha tenido un leve impacto incremental en la mayoría de estados miembros, algo que fue evaluado como un éxito al no esperarse nada más razonable de dicho mecanismo. La evaluación, sin embargo, muestra que el instrumento no funciona exactamente tal y como estaba previsto que lo hiciese.

Palabras clave Evaluación europea, Método Abierto de Coordinación.

\section{An Evaluation of the Soft Coordination approach of the European Governments' Policies}

\begin{abstract}
This paper derives from an evaluation carried out by the author and several of his colleagues (From Euréval and Ramboll Management) for the European Commission in 2007. The evaluation applied to the coordination of national policies in the areas of economic growth and employment. The main conclusion of the report (<http://ec.europa.eu/economy finance/>) is that the soft coordination approach is effective in fostering mutual learning, enlarging consensus among stakeholders, reinforcing reform promoters' legitimacy, and finally pushing reforms upward on the political agendas. The instrument has had a small incremental impact in a majority of Member States, something which was assessed as a success since nothing more could reasonably be expected from such a soft mechanism. The evaluation however shows that the instrument does not work exactly as it was assumed to work.
\end{abstract}

Key words

European Evaluation, Open Method Coordination. 
Nacida de la experiencia adquirida en las áreas de asuntos económicos y empleo, la "coordinación suave" (soft coordination) de las políticas nacionales europeas cobró impulso en la década de 1990 bajo la denominación de "Método Abierto de Coordinación (MAC)" (Hodson et al., 2001). Esta aproximación se sustenta en instrumentos blandos tales como la creación de consenso, el aprendizaje mutuo, la participación de los interesados y la coordinación de las agendas de reforma nacional. Continuos debates han cuestionado el papel de instrumentos más duros tales como "señalar con el dedo", estándares (benchmarks) cuantitativos, tablas de rankings (league tables), revisiones inter pares (peer-review) y recomendaciones, señalando los fallos de algunos Estados miembros.

En 2005, los dirigentes de la UE decidieron mejorar los procesos de coordinación y se modificó el mecanismo de coordinación para aclarar responsabilidades, promover la apropiación (ownership) en los Estados miembros e integrar el conjunto de objetivos de una política en un paquete simplificado de "Directrices Integradas para el Crecimiento y el Empleo", en adelante, DICE.

En 2007, la Comisión Europea inició una evaluación de dicha reforma, con vistas a una perspectiva más amplia de la coordinación de los Estados miembros durante los últimos diez años. Dicha evaluación externa fue dirigida por la Comisión y abarcó una serie de actividades de coordinación que estaban bajo su responsabilidad, al menos en parte. Tenía un fin formativo y fue ideada para proponer mejoras tanto en el contenido de las directrices como en el funcionamiento del proceso de coordinación.

La principal conclusión de este informe fue que la aproximación de la "coordinación suave" es eficaz para fomentar el aprendizaje mutuo, incrementar el consenso entre las partes interesadas, reforzar la legitimidad de los promotores de la reforma y, finalmente, para darles prioridad a las reformas en las agendas políticas. Sin embargo, este instrumento sólo tiene un pequeño impacto marginal en la mayoría de los Estados miembros, algo que se consideró un éxito dado que no se podía esperar mucho más de un mecanismo tan blando. Esta evaluación, sin embargo, muestra que el instrumento no funciona precisamente como se esperaba (véase el Apartado 4.1).

Este artículo es fruto del informe de evaluación ${ }^{1}$ que fue redactado por el autor y varios de sus colegas². Las páginas que siguen desarrollan los siguientes puntos en orden sucesivo:

- La evaluación y el enfoque metodológico aplicado para abordar las cuestiones de causa y efecto, es decir, el "análisis de contribución".

1 Evaluación de las Directrices Integradas para el Crecimiento y el Empleo. En línea: <http:// ec.europa.eu/economy_finance/evaluation/pdf/final_report_ipg_en.pdf.>.

2 De Euréval y Rambøll Management. 
- El proceso de "coordinación suave" y las presunciones subyacentes de causa y efecto.

- Los principales resultados y conclusiones.

\section{LA EVALUACIÓN}

\subsection{Cuestionando la eficacia de la coordinación}

La evaluación se inició dos años después de la reforma del 2005 y un año antes de que se tuviera que revisar el instrumento de coordinación. En los términos de referencia de la evaluación, la Comisión Europea realizó ocho preguntas, dos de las cuales se referían a la eficacia de la "coordinación suave". Este artículo se centra en dos de estas preguntas:

- ¿Ha ayudado el instrumento de "coordinación suave” a plantear temas que de otra manera no se habrían abordado?

- ¿Ha ayudado el instrumento de "coordinación suave” a avanzar en la agenda de reforma a nivel nacional y comunitario?

Responder a las preguntas anteriores requiere un análisis de las causas y efectos en un contexto desafiante por varias razones. En primer lugar, el instrumento de coordinación se aplica de una manera muy flexible a través de agendas de reformas nacionales que difieren significativamente entre sí. En segundo lugar, el instrumento se aplica tanto en países grandes como pequeños, federales o unitarios, familiarizados con los asuntos de la UE y recién Ilegados. En tercer lugar, la coordinación no puede afectar a las políticas nacionales a través de unos mecanismos de causa y efecto lineales y simples. Al contrario, los efectos deseados cobran forma tras una cadena de múltiples efectos, en un buen número de partes interesadas, la mayoría involucradas en sistemas abiertos y amplios. En cuarto y último lugar, todas las causas y efectos son intangibles y por tanto es casi imposible basarse en hechos concretos.

En este contexto, habría resultado imposible aplicar cualquier tipo de razonamiento contrafactual (¿qué habría ocurrido en la política de un Estado miembro de no existir el mecanismo de "coordinación suave" de la UE?). Incluso el concepto de "evidencia" se vuelve cuestionable.

\subsection{Utilizando el análisis de contribución}

El equipo de evaluación afrontó los retos anteriormente mencionados mediante un método nuevo que se basa, inter alia, en el "análisis de contribución" y en el estudio de casos embebidos. 
El análisis de contribución (Mayne, 1999; Mayne, en prensa) se introdujo en 1999. Aunque se ha discutido ampliamente a lo largo de la década pasada, los casos prácticos de implementación siguen siendo escasos en la literatura. La aproximación procede siguiendo los pasos sucesivos que se detallan a continuación:

- Delinear el/los asunto/s de causa y efecto y generar una o varias cadenas de suposiciones causales.

- Recolectar datos primarios e información secundaria con el propósito de cubrir todas las cadenas de causa y efecto en toda su dimensión. No es un problema si los datos son cualitativos o cuantitativos. Más bien al contrario, lograr un equilibrio entre los hechos y las opiniones que confirmen o nieguen las suposiciones causales es de gran importancia para evaluar la credibilidad de toda la información.

- Escribir un borrador mostrando la interpretación del analista de la información completa (el borrador "historia de contribución”). Este texto sigue las cadenas de suposiciones causales siguiendo un sistema "paso-a-paso". En cada fase, el analista evalúa (1) si el cambio o evento pretendido ocurrió, y (2) qué factores contribuyeron a ello y cuáles fueron sus papeles respectivos para ilustrar lo que pasó (o no pasó).

- El borrador se somete a una crítica sistemática fortaleciendo la información base y el análisis hasta proporcionar una historia de contribución final y robusta.

Esta aproximación es parte de la familia de métodos de evaluación "de base teórica”. Presenta como novedad la realización de todas las hipótesis sucesivas evaluadas de una forma sistemática y organizada. Una de sus fortalezas es que es factible en muchos entornos, tanto en los simples como en los complejos. Cabe recordar que el análisis de contribución no requiere de ningún razonamiento contrafáctico.

Una de las primeras partes de los trabajos consistió en construir un modelo lógico para mostrar las hipótesis prevalecientes sobre causas y efectos (véase el Apartado 2.4 y la Figura 1). La historia de contribución final se resume al final de este artículo (véase el Apartado 4.1 y la Figura 2). La opinión del autor es que la aproximación metodológica aplicada en este difícil contexto ha hecho que sea posible responder a las preguntas de un modo claro y directo, incluso en ausencia de hechos concretos y de razonamiento contrafáctico.

\subsection{Recogida de datos primarios mediante estudio de caso}

Esta evaluación se basó en una serie de fuentes de información que se recogie56 ron y/o produjeron entre los meses de julio y septiembre de 2007: 
- Análisis documental (documentos oficiales, evaluaciones anteriores, trabajos de los expertos);

- Bases de datos de la Comisión Europea;

- Entrevistas exploratorias en la Comisión, el Parlamento Europeo y algunos Estados miembros;

- Hojas de datos describiendo las disposiciones implementadas por los Estados miembros para gestionar el proceso de coordinación;

- Entrevistas semi-estructuradas con los responsables de las políticas, los expertos y los interesados a través de un enfoque "bola de nieve" en 18 países (70 entrevistas);

- Casos de estudio cubriendo cuatro áreas políticas y trece Estados miembros.

Entre los recursos mencionados, los casos de estudio resultaron ser los más importantes para sugerir y apoyar las conclusiones del análisis. Se organizaron en el formato de "casos de estudio embebidos" en dos niveles, es decir, (1) áreas políticas y (2) Estados miembros. Al principio se seleccionaron cuatro casos en función de cuatro ámbitos políticos en los que los compromisos de reforma conjuntos se hicieron en toda Europa. Entonces, en cada caso principal se profundizó en una serie de cuatro sub-casos, es decir, se seleccionaron cuatro Estados miembros por sus conocidos logros, buenos o malos, en el ámbito correspondiente. El equipo de evaluación entonces disponía de dieciséis oportunidades para analizar el impacto del instrumento de coordinación sobre la realización de políticas. En lo sucesivo, llamaremos a estos análisis "estudios de países". En algunos casos, se investigaron dos áreas de políticas en un mismo Estado miembro, y esto se traduce en el hecho de que hubiera dieciséis sub-casos en las investigaciones en las que solo había trece Estados miembros involucrados 3 .

Incorporarle a los casos de estudios dos o más niveles de análisis es una herramienta muy poderosa para recoger el tipo de información necesaria en el contexto de un análisis de contribución. Si tales casos de estudio están debidamente diseñados, pueden llegar a cubrir las cadenas de causa y efecto en toda su longitud y proporcionar, de tal modo, un material lo suficientemente rico para ser procesado por una analista.

En general, se puede afirmar que un método basado en casos de estudio embebidos y el análisis de contribución ha posibilitado que el equipo de análisis

3 España no se analizó por motivos meramente prácticos. No hay ningún indicio de que España hubiera implementado el proceso de "coordinación suave" mejor o peor que los demás Estados miembros. 
responda a una serie de preguntas difíciles, que habrían sido difíciles de afrontar de una manera concluyente y convincente mediante las aproximaciones tradicionales.

\section{EL PROCESO DE “COORDINACIÓN SUAVE”}

Las páginas siguientes desarrollan en orden sucesivo:

- El origen y la naturaleza del instrumento de coordinación;

- El proceso de coordinación actual;

- La lógica de los efectos previstos.

\subsection{Origen y contenido de las directrices}

Las raíces del instrumento de "coordinación suave" se pueden trazar hasta, al menos, la publicación de la "estrategia de cooperación para el crecimiento y el empleo" a mediados de 1992 y la consiguiente primera edición de las Orientaciones Generales de las Políticas Económicas de 1993 (OGPE). El Tratado de Ámsterdam de 1997 otorgó al Consejo la competencia para establecer unas recomendaciones sobre las cuales los Estados miembros estaban obligados a actuar en el ámbito de las políticas de empleo. En ese mismo año, una cumbre especial en Luxemburgo desarrolló el primer conjunto de Directrices para el Empleo (DE).

En los años siguientes la OGPE y las Directrices de Empleo estuvieron sometidas a procesos de coordinación separados. Las políticas económicas y de empleo se combinaron por primera vez en la "Estrategia de Lisboa"4 en 2000, junto con una dimensión medioambiental. En 2004, una revisión ${ }^{5}$ de la Estrategia de Lisboa criticó la implementación del instrumento de coordinación en los siguientes términos: "Los Estados miembros no asumen [la Estrategia de] Lisboa y la Comisión no está preparada para identificar y culpar a los que yerran".

En el Consejo Europeo de primavera de 2005, los dirigentes de la UE decidieron renovar la Estrategia de Lisboa para el período 2005-2008 y mejorar los mecanismos de coordinación. Los cambios principales fueron:

- Una nueva estructura de gobierno con responsabilidades más claras; 
- Una mayor atención a la asociación y la menor dependencia de los indicadores y las tablas de rankings, con el fin de promover la apropiación en los Estados miembros;

- Un conjunto de directrices menor (24 en lugar de unas 100) y la agrupación de las directrices cubriendo todos los ámbitos políticos en un "paquete integrado";

- Un proceso de coordinación único que fusiona los anteriores, aunque se mantengan las distinciones en las medidas legales en los diferentes ámbitos políticos.

El Paquete Integrado de Directrices se compone de tres grupos (asuntos microeconómicos, macroeconómicos y empleo) y cada uno consta de entre seis y diez directrices. Una directriz describe entre una a cinco clases de reformas propuestas para un ámbito político determinado. En conjunto, se describen 68 clases de reformas de una forma relativamente amplia (por ejemplo, "reforzar las pensiones, el seguro social y los sistemas sanitarios para garantizar que son viables económicamente y que son socialmente adecuadas y accesibles"). Cada directriz tiene una breve introducción justificando las reformas en términos de sus aportaciones al crecimiento y el empleo.

La sexta directriz se dedica a la zona Euro y tiene una naturaleza especial ya que no recomiendan reformas específicas sino que ejerce presión para que se "continúe" la implementación activa de las otras 23 directrices en los países de la zona Euro.

La mayoría de las directrices se expresan en términos de reformas, con la excepción de la directriz 17, que se expresa como un objetivo: "para lograr una tasa media de empleo del $70 \%$ en 2010".

\subsection{Procedimiento de coordinación}

El proceso de coordinación se divide en períodos de tres años. La evaluación se aplica al período que comenzó con la aprobación de las directrices en junio de 2005. El primer paso fue la formulación del Programa Nacional de Reformas (PNR) en octubre de 2005. Este proceso se reflejó a nivel europeo con el Programa Comunitario de Lisboa adoptado en junio de 2006. Los siguientes pasos se sucedieron mediante ciclos anuales.

En el mes de octubre de cada año los Estados miembros entregan su Informe de Implementación que se debate en una serie de reuniones de "supervisión multilateral”. Seguidamente, la Comisión prepara su informe anual de progresos y un borrador 


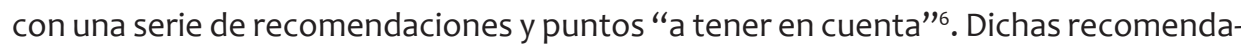
ciones se debaten con los representantes de los Estados miembros, bilateralmente o en reuniones del Comité. Seguidamente, el informe y el borrador de las recomendaciones son examinados por las Comisiones Parlamentarias y se someten al Consejo para su aprobación en las reuniones de primavera. Las recomendaciones se citan y comentan en los medios de comunicación.

Los Estados miembros han designado "Coordinadores Nacionales de Lisboa" que mantienen contactos a nivel ministerial al menos tres veces al año. La mayoría de los Estados miembros tienen un comité/grupo exclusivamente dedicado al instrumento de "coordinación suave". Tales grupos se suelen reunir tres veces al año o incluso más.

Además de este proceso de coordinación estructurada, la Comisión y/o los Comités del Consejo organizan ad hoc grupos de trabajo, revisiones inter pares y sesiones de aprendizaje mutuo ${ }^{7}$. En el ámbito del empleo, dichas actividades se sustentan en el Programa para el Aprendizaje Mutuo ${ }^{8}$ que incluye (1) seminarios semestrales temáticos a escala europea sobre los principales desafíos o prioridades políticas, (2) reuniones de revisión inter pares en Estados miembros individuales sobre medidas y políticas específicas, y (3) actividades de seguimiento y difusión involucrando a un grupo más amplio de actores implicados a nivel nacional.

\subsection{La lógica de los efectos previstos}

Este apartado explica cómo el instrumento de "coordinación suave" fue asumido para obtener los efectos deseados. La Figura 1 muestra una serie de hipótesis lógicas identificadas por el equipo de evaluación al leer los documentos básicos y las entrevistas iniciales. Una docena de versiones sucesivas de esta figura fueron desarrolladas por el equipo de evaluación hasta que se llegó a un acuerdo satisfactorio entre: (a) una exposición fiel de los documentos y de las entrevistas iniciales, (b) coherencia conceptual, y (c) simplicidad. Las hipótesis se muestran siguiendo un orden lógico que no es necesariamente cronológico.

Los objetivos fundamentales de la Estrategia de Lisboa eran convertir a Europa en un lugar más atractivo para invertir y trabajar, el crecimiento basado en el conoci-

6 En 2006 se realizaron 50 recomendaciones (una media de dos por cada Estado miembro) y 133 puntos de seguimiento (cinco por Estado miembro, de media). En adelante el término "recomendación" se usará en el sentido más amplio, incluyendo también los puntos de seguimiento.

7 Algunos servicios de la Comisión publican indicadores y tablas de rankings, por ejemplo el Esquema Orientativo sobre la Innovación (http://trendchart.cordis.europa.eu/), pero tales actividades no son objeto de esta evaluación.

8 Iniciado a principios de 2005 como seguimiento del Programa de Evaluación de Homólogos de 
miento y la innovación, y la creación de más y mejores empleos. La justificación para una intervención a nivel comunitario se recogió en el texto de la Estrategia de Lisboa de la siguiente manera: “Las economías europeas son interdependientes. La prosperidad en un Estado miembro crea prosperidad en los demás. La desidia de un Estado miembro retiene a los demás. Los Estados miembros deben considerar sus políticas económicas como una cuestión de interés común".

FIGURA 1

La lógica general del instrumento de "coordinación suave"

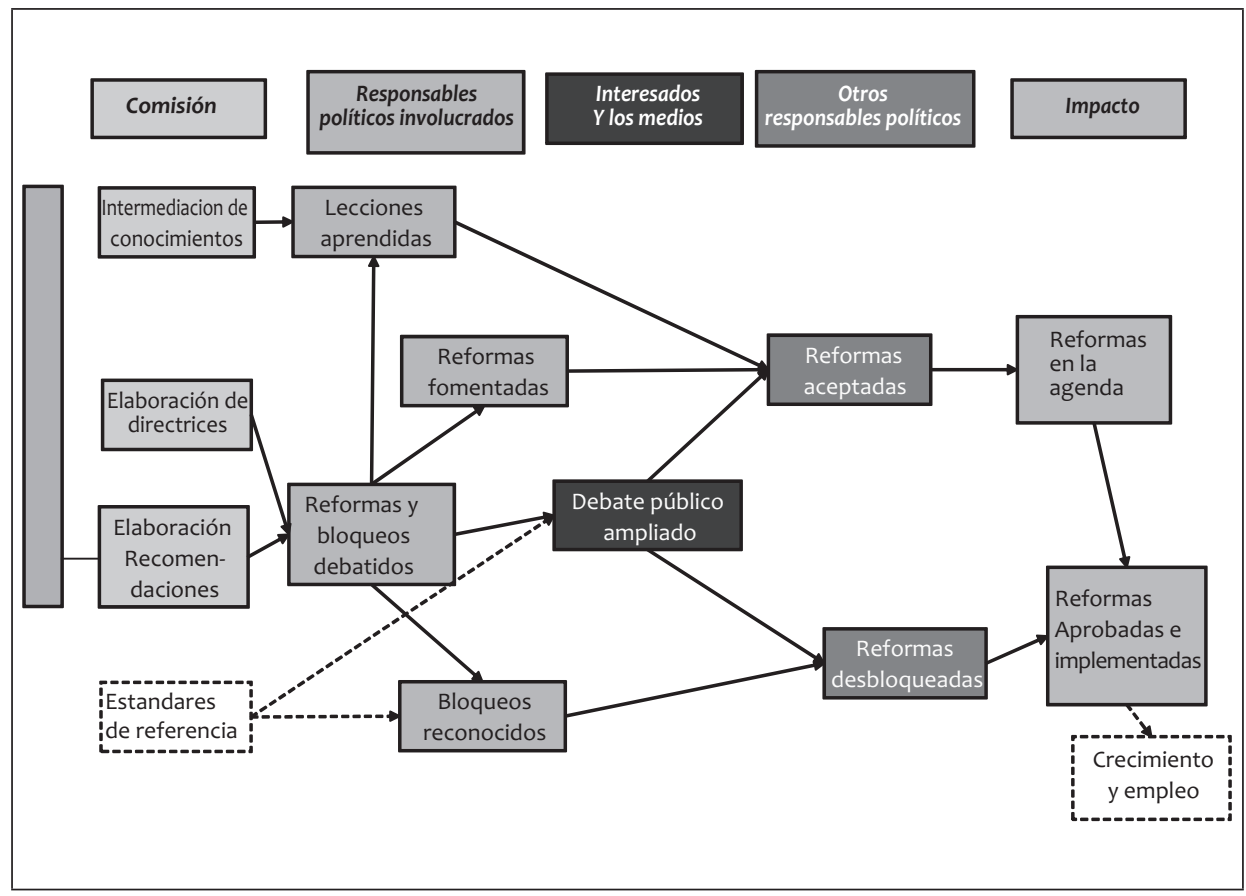

Fuente: Equipo de evaluación. Las flechas representan las hipótesis causa-efecto. Los recuadros y las flechas de puntos no se reutilizaron en el análisis de causalidad.

Los objetivos estratégicos se deben obtener mediante un instrumento de "coordinación suave” principalmente porque las alternativas son política o pragmáticamente inaceptables. La gran ventaja del instrumento es que permite hacer algunas políticas comunes sin la necesidad de aplicar disposiciones legales vinculantes o realizar una trasferencia completa de la competencia a un nivel supranacional, con todas las complicaciones que ello conlleva. El instrumento también incorpora la suficiente flexibilidad para adaptarse rápidamente, al contrario que las disposiciones legales vinculantes, y para tener suficientemente en cuenta las diferencias nacionales" (Begg, 2006: 56). 
Los principales efectos de los instrumentos de coordinación son aplicables a los responsables políticos implicados, pero la agenda de reformas y la aprobación de las reformas están principalmente bajo el control de otros responsables políticos que están más involucrados en la política interna. Los "responsables políticos involucrados" son los oficiales de la Comisión Europea responsables del sistema de coordinación, los coordinadores nacionales de Lisboa y los representantes de los Estados miembros involucrados en actividades de coordinación.

El impacto sobre el crecimiento y el empleo se muestra con líneas de puntos para mostrar que no han sido objeto de la evaluación. Lo mismo se aplica a las actividades de la Comisión relativas a los indicadores, tablas de rankings y la evaluación comparativa. Estas actividades se enmarcan en algunos ámbitos de políticas como continuación del anterior “Método Abierto de Coordinación". Ya no son parte de la lógica del instrumento de "coordinación suave" tras su reforma en 2005 .

Por último, integración y complementariedad deben entenderse como dos principios transversales que se aplican (o deberían aplicarse) a todo el proceso. La integración se entiende como el hecho de que una u otra reforma cubra la totalidad de las cuestiones en juego, incluyendo la incidencia negativa sobre los que podrían salir perdiendo con la reforma, minimizando así las pérdidas y/o compartiéndolas. La complementariedad se entiende como el hecho de que las reformas de un Estado miembro y la Comunidad se complementan y refuerzan entre sí, cada nivel de Gobierno se centra en las áreas en las que aporta un valor añadido.

\section{PRINCIPALES RESULTADOS Y CONCLUSIONES}

\subsection{El instrumento de "coordinación suave" funciona}

\subsubsection{La "coordinación suave" puede ser parte de un "policy mix" (combinación de instrumentos de políticas)}

El equipo de evaluación estima que aproximadamente 50\% de las reformas previstas se afrontan mediante el instrumento de "coordinación suave" solamente, o mediante instrumentos obligatorios (4\%), programas de gastos (26\%), o regulación dura (20\%).

Dos de los cuatro casos estudiados incluyen tal combinación de políticas (véase el Cuadro 1). El caso del aprendizaje permanente muestra una estrategia muy coherente en la que el instrumento de "coordinación suave" se usa para aprovechar e incrementar parte de las asignaciones del Fondo Social Europeo para las reformas prioritarias. En el caso de las profesiones reguladas se sugiere que puede haber una sinergia a largo plazo entre el uso actual de la "coordinación suave" y una política 62 más dura en el futuro. 


\section{CUADRO 1}

"Policy mix" en dos áreas: el aprendizaje permanente y las profesiones reguladas

El asunto del aprendizaje permanente se ha desarrollado progresivamente desde el Libro Blanco acerca de la enseñanza y el aprendizaje publicado en 1995. En aquellos momentos, el asunto de la "adaptabilidad" también estaba sujeto a un gran debate en el contexto del Fondo Social Europeo (FSE). El FSE es, por supuesto, un instrumento de política dura en comparación con las directrices.

A lo largo de los últimos diez años, el asunto de la enseñanza desde el pre-escolar hasta la edad adulta se ha popularizado y los dos instrumentos (Lisboa y FSE) se han ido combinando progresivamente. Cabe destacar en esta evaluación los ejemplos de Estonia - que ha mostrado un gran interés en aprender de Irlanda cómo usar las ayudas del FSE para promover el aprendizaje permanente; y de Grecia - que ha destinado un tercio de la asignación de la FSE al aprendizaje permanente.

El asunto de las profesiones reguladas se aborda en el marco de las políticas de competencia, que ha sido una competencia Comunitaria desde 1957 y se enmarca dentro de la estrategia del Mercado Único desde 1993. La influencia europea sobre las políticas de competencia nacionales ha aumentado en los últimos años gracias a una serie de impulsos de la Comisión (grandes informes de la Dirección General en 2004 y 2005 y comunicados sobre la compatibilidad entre la legislación de la competencia Comunitaria y las normas que regulan las profesiones), las resoluciones del Parlamento Europeo sobre varias profesiones (salud, derecho, contabilidad, etc.) y directivas acerca del mutuo reconocimiento de las cualificaciones profesionales y la libre circulación de servicios en toda Europa. Aparte de la transposición de servicios de la Directiva, los Estados miembros están obligados a constituir autoridades de competencia nacionales eficaces e independientes.

El poder regulador a escala comunitaria en este ámbito sigue siendo incierto ya que no hay evidencias de que su desarrollo esté conectado al proceso de Lisboa. El instrumento de "coordinación suave" se podría usar para popularizar un asunto que será abordado mediante algún tipo de regulación dura en el futuro. Esta hipótesis, sin embargo, es cuestionada por el hecho de que las reformas sobre las profesiones reguladas son algunas de las que menor éxito tienen.

Fuente: Equipo de evaluación.

\subsubsection{Conformación de las agendas de reformas.}

En el marco de la encuesta del equipo de evaluación, cerca del 80\% de los encuestados dijeron que "los responsables políticos están comprometidos a fomentar el uso de las directrices en sus respectivos países”. ¿Acaso significa esta declaración que los Estados miembros apoyan el proceso de coordinación o es solo una retórica para cumplimiento? Probablemente haya algo de verdad en las respuestas a este cuestionario dado que las respuestas de los responsables políticos involucrados (aquéllos que asisten a las reuniones en Bruselas) y las de los demás interesados (a los que se les supone menos dados al uso de la retórica del cumplimiento) son parecidas. 
Como se muestra en la Tabla 1, un número de estudios de países confirman que las directrices dan forma a los debates políticos y al modo en el que se preparan las reformas, incluso cuando su influencia sobre la aprobación en sí de las reformas es limitada. Esta tabla y las siguientes resumen los resultados de los dieciséis "estudios de países" (sub-casos) desarrollados en el marco de esta evaluación.

\section{TABLA 1}

Agendas de reformas: demostrando la hipótesis ${ }^{9}$

\begin{tabular}{|c|c|c|c|c|}
\hline Envejecimiento & $\begin{array}{c}\text { Mejor } \\
\text { regulación }\end{array}$ & $\begin{array}{c}\text { Profesiones } \\
\text { Reguladas }\end{array}$ & $\begin{array}{c}\text { Aprendizaje } \\
\text { permanente }\end{array}$ & $\begin{array}{c}\text { Hipótesis } \\
\text { confirmada }\end{array}$ \\
\hline $\mathrm{DE}$ & $\mathrm{UK}$ & $\mathrm{GR}$ & $\mathrm{DK}$ & Sí \\
\hline $\mathrm{OS}$ & $\mathrm{NL}$ & $\mathrm{IT}$ & $\mathrm{EE}$ & Más bien sí \\
\hline $\mathrm{SL}$ & $\mathrm{DE}$ & $\mathrm{IE}$ & $\mathrm{NL}$ & Más bien no \\
\hline $\mathrm{PT}$ & $\mathrm{PL}$ & $\mathrm{FR}$ & $\mathrm{GR}$ & No \\
\hline \multicolumn{4}{|r}{} \\
\cline { 3 - 5 }
\end{tabular}

Fuente: Equipo de evaluación.

Por ejemplo, en el caso del aprendizaje permanente, tanto Estonia como Grecia diseñaron sus nuevas estrategias basándose en las directrices. En ambos países, el término "aprendizaje permanente" y el indicador asociado se volvieron públicamente visibles en aquel momento y ahora ambos son parte del debate público (véase el Cuadro 2).

El caso del envejecimiento es una historia de éxito en la conformación de los debates políticos. Por toda Europa, el asunto se discute en referencia a los conceptos y los indicadores desarrollados desde finales de la década de 1990 en el contexto del proceso de "coordinación suave", por ejemplo "la incidencia del envejecimiento en porcentaje del Producto Interior Bruto en 2050", y “el porcentaje de personas por encima de los 55 con trabajo remunerado". Se hacen referencias sistemáticas a esta forma de pensar en los medios de comunicación, incluso si no se citan las directrices o los procesos de coordinación.

En general, la idea es que los responsables políticos tienden a fomentar las reformas políticas en sus países, no solo porque están de acuerdo con las directrices y tienen un sentimiento de apropiación, sino principalmente porque piensan en las reformas en los términos y con la mentalidad asociada con las directrices.

9 La hipótesis analizada era la siguiente: mediante su involucración en la discusión acerca de las directrices y recomendaciones, los responsables políticos que participan en el proceso son animados a fomentar las reformas. Entonces, las reformas que son ampliamente aceptadas en un círculo más amplio de 64 responsables políticos, suben en la agenda y finalmente son aprobadas e implementadas (véase el Aparta- 


\section{CUADRO 2}

\section{El asunto del aprendizaje permanente en Estonia y Grecia}

Estonia aprobó la Ley de Enseñanza para Adultos en 1993 en virtud de la cual se establecía el derecho a la enseñanza durante la vida profesional y para establecer las responsabilidades de los empresarios y el gobierno central y local en este asunto. El asunto reapareció en la agenda política en 2005 cuando la estrategia de Estonia del Aprendizaje Permanente fue aprobada, junto con una reforma de los sistemas de financiación por la que se comparten los gastos de formación entre los empresarios, los empleados y el Estado. El Fondo Social Europeo se ha identificado como uno de los elementos claves para la implementación exitosa de dicha reforma.

Está claro que los debates políticos se han visto modelados por el indicador utilizado durante del proceso de coordinación (porcentaje de la población adulta en edad de trabajar que ha participado en alguna actividad de formación en las últimas cuatro semanas). Estonia sigue estando lejos de la media de la UE, del objetivo del $12,5 \%$ y también de su propia meta nacional del $10 \%$.

La reforma también se debe a una aportación de la Comisión que, en una reunión de preparación de PNR en Tallin en 2005, mostró la necesidad del aprendizaje permanente. La reunión contó con la asistencia de los ministros, parlamentarios y los representantes de los interlocutores sociales. Los comentarios de la Comisión fueron procesados entre otros en un grupo de trabajo incluyendo varios ministerios (Asuntos Sociales, Economía y Hacienda), interlocutores sociales y expertos académicos.

Estonia participó en el Comité de Educación en el grupo de trabajo del programa Educación y Formación 2010, donde conoció la práctica irlandesa de utilizar los Fondos Estructurales para fomentar la formación continua.

Desde el principio de los noventa hasta el 2003, Grecia desarrolló progresivamente su sistema de formación profesional, obteniendo unos resultados limitados. Destinó cerca de un tercio de los fondos del FSE del período 2000-2006 a acciones de formación profesional permanente. En el 2005, el gobierno aprobó una nueva ley cuya intención específica era alcanzar un objetivo de 12,5\% de participación de la población activa en formación permanente para el 2010.

La nueva agenda de Lisboa era el marco para esta iniciativa y apoyó las iniciativas políticas del Gobierno en este ámbito. Sin embargo, debido a las elecciones generales de septiembre de 2007 y a la falta de tradición de coordinación social, no se ha implementado ninguna iniciativa significativa hasta el momento.

Las autoridades griegas han desarrollado un papel importante en las actividades de aprendizaje en el marco de Educación y Formación 2010. El Reino Unido destaca como el país del que Grecia más quiere aprender, no sólo porque se ve como uno de los países que obtiene buenos resultados en Europa, sino porque muchos griegos han estudiado en el Reino Unido y mantienen contactos permanentes con este país.

Fuente: Equipo de evaluación.

\subsubsection{Aprendizaje mutuo}

La Tabla 2 resume los resultados de los 16 estudios de países y muestra que el aprendizaje mutuo ha ocurrido de forma sistemática, al menos en una pequeña medida. 
TABLA 2

Aprendizaje mutuo: demostrando la hipótesis ${ }^{10}$

\begin{tabular}{|c|c|c|c|c|}
\hline Envejecimiento & $\begin{array}{c}\text { Mejor } \\
\text { regulación }\end{array}$ & $\begin{array}{c}\text { Profesiones } \\
\text { Reguladas }\end{array}$ & $\begin{array}{c}\text { Aprendizaje } \\
\text { permanente }\end{array}$ & $\begin{array}{c}\text { Hipótesis } \\
\text { confirmada }\end{array}$ \\
\hline $\mathrm{DE}$ & $\mathrm{UK}$ & $\mathrm{GR}$ & $\mathrm{DK}$ & Sí \\
\hline $\mathrm{OS}$ & $\mathrm{NL}$ & $\mathrm{IT}$ & $\mathrm{EE}$ & Más bien sí \\
\hline $\mathrm{SL}$ & $\mathrm{DE}$ & $\mathrm{IE}$ & $\mathrm{NL}$ & Más bien no \\
\hline $\mathrm{PT}$ & $\mathrm{PL}$ & $\mathrm{FR}$ & $\mathrm{GR}$ & No \\
\hline \multicolumn{4}{|r}{} & ? \\
\cline { 4 - 5 } & &
\end{tabular}

Fuente: Equipo de evaluación.

Cerca del 70\% de los encuestados (principalmente responsables políticos involucrados - véase el apartado 1.3) confirmaron que había habido aprendizaje mutuo. Las afirmaciones estaban relativamente equilibradas entre "enseñando a otros" y "aprendiendo de otros". El aprendizaje parece haber sido más importante en el ámbito de las políticas sociales.

Los participantes aprendieron nuevos conceptos, prácticas interesantes e historias citables para dotar de más legitimidad a las reformas y hacerlas más aceptables para los líderes de opinión y los ciudadanos.

Los encuestados explicaron qué aprendieron principalmente en las reuniones del comité y en los contactos con los coordinadores nacionales de Lisboa. Algunos encuestados aprendieron del material preparado para las reuniones del comité. La interacción se cita con mayor frecuencia como un canal de aprendizaje, por ejemplo; "momentos informales durante las conferencias de revisión entre homólogos son cruciales para el aprendizaje”.

En general, está claro que el proceso de "coordinación suave" contribuye al aprendizaje, primero porque la Comisión transmite ejemplos de buenas prácticas y, en segundo lugar, porque los grupos de trabajo inician conexiones entre los Estados que enseñan y los que aprenden, es decir, entre los precursores y los Estados que han quedado rezagados. Sin embargo, hay muchos otros canales de aprendizaje, la mayor alternativa son las redes de expertos.

\subsubsection{Primera conclusión: El instrumento de "coordinación suave" funciona}

El estudio confirma la hipótesis de que el instrumento de "coordinación suave" es eficaz para dar forma a la agenda de reformas y para fomentar el aprendizaje mu-

10 La hipótesis analizada era la siguiente: el debate acerca del borrador de directrices y recomendaciones, además de los esfuerzos de la Comisión por transferir conocimientos conllevan a reformas que 66 das e implementadas (véase el Apartado 2.3 y la Figura 1). 
tuo. A la vista de la información recogida y el posterior análisis, se puede decir que el instrumento tiene un impacto incremental sobre las agendas de reformas en la mayoría de los Estados miembros ${ }^{11}$.

¿Se debería considerar suficiente este impacto? La definición del umbral del éxito es un asunto complicado que se debatió extensamente en el grupo de dirección del estudio. No hay puntos de referencia disponibles ya que los instrumentos de "coordinación suave" no se han analizado extensamente hasta ahora. En consonancia con la práctica de evaluación de la OCDE (OCDE 2007), que decidió que el sistema de "coordinación suave" podía ser analizado positivamente si se llegaran a acelerar y mejorar los procesos de reforma en la mayoría de los Estados miembros, incluso marginalmente (véase el Cuadro 3).

\section{CUADRO 3 \\ ¿Qué es una "coordinación suave" exitosa?}

En los (escasos) análisis de sistemas intergubernamentales similares, se aplicaron los siguientes umbrales de éxito: (1) mitad o más de los miembros califican a un objetivo político como altamente relevante, (2) dos tercios o más de los miembros califican el resultado como de alta calidad, (3) más de la mitad de los miembros califican el instrumento de tener una contribución media en términos de reestructuración sostenible de las políticas nacionales.

Este análisis se refiere principalmente a inducir las reformas políticas. El equipo de evaluación y el equipo de dirección, por tanto, han acordado considerar como éxito el hecho de que el proceso de las DICE (Directrices Integradas para el Crecimiento y el Empleo) hayan afectado las agendas de reformas europeas tan ampliamente; se aplica el umbral de "la mitad" para determinar la amplitud de la influencia.

La elaboración de políticas es el resultado de un complejo abanico de fuerzas nacionales e internacionales. En este contexto, la profundidad de la influencia que puede ser ejercida por un instrumento de "coordinación suave" es inevitablemente limitada. Esta evaluación ha adoptado la opinión de que la "coordinación suave" puede considerarse como exitosa, incluso si ha tenido una influencia marginal sobre la agenda de reformas (por ejemplo, mejorando la calidad) en lugar de por la introducción de nuevas reformas.

La velocidad también es una dimensión importante del éxito dado que "el tiempo es la esencia de la credibilidad de la Estrategia de Lisboa" (Sapir, 2007). No obstante, debemos reconocer que la reforma de una amplia gama de políticas en toda Europa es un proceso que no puede ser otra cosa que largo. Por ejemplo, el despliegue actual del concepto de "Flexiguridad" tiene diez años pero aún no está completamente maduro. ¿Cómo pueden ajustarse la perspectiva a medio plazo de las directrices con su ciclo de tres años y el proceso a largo plazo de cambiar la mentalidad y las instituciones de los países? Este análisis ha adoptado la perspectiva de que el éxito de las DICE tienen mucho que ver con la aceleración de las reformas en curso.

Fuente: Equipo de evaluación.

11 Esta valoración no está en línea con el Informe elaborado por I.Begg: "Sin embargo, si es para proporcionar valor añadido a lo que las políticas nacionales obtendrían en su ausencia, el MAC también debe englobar la forma de cambiar lo que harían los Estados miembros en cualquier caso y no es, de ninguna forma, evidente que lo logre el marco de Lisboa II" (Parlamento Europeo, 2006: 56). 
En referencia a este umbral, se consideró que el instrumento de "coordinación suave" estaba funcionando lo suficientemente bien.

\subsection{El instrumento no funciona como se pretendía}

Al comienzo de este análisis se supuso que el instrumento "suave" de política ejerce una presión sobre los gobiernos y contribuye, incluso marginalmente, al desbloqueo de las reformas políticas (véase la Figura 1). Este apartado define el término "presión" (véase el Cuadro 4) y muestra en qué sentido, el instrumento no funcionó como se esperaba.

\section{CUADRO 4}

\section{El asunto del envejecimiento en Portugal y Eslovenia}

El proceso de reformar el sistema de pensiones en Portugal dio un primer paso en el año 2000, con una ley que prevé el nuevo modelo económico, un fondo de reserva y un nuevo régimen de pensiones.

El año 2005 fue un punto de inflexión: el gasto público estaba en su nivel más alto, se eligió un nuevo gobierno en marzo, se revisó el Pacto de Sostenibilidad y Crecimiento y se publicó el DICE. Se constituyó un comité compuesto por los representantes de los Ministerios de Economía y Trabajo para valorar el impacto del envejecimiento de la población desde una perspectiva a largo plazo y para evaluar medidas específicas. Se diseñaron dos grandes reformas: el Nuevo Plan de Empleo Nacional (2005) y la Estrategia Nacional Activa de Envejecimiento (2006) incluyendo incentivos para permanecer en el mercado laboral.

Portugal se ha convertido en un participante activo en los mecanismos de "coordinación suave", y esto se dice que es "un elemento de presión para elevar los principales problemas y para hacer esfuerzos para superarlos". Los factores clave para explicar las reformas, sin embargo, han sido el cambio de gobierno, el hecho de que los agentes sociales y la opinión pública eran conscientes del estado de emergencia del sistema social y el Pacto de Estabilidad y Crecimiento.

En Eslovenia el sistema de pensiones estuvo en la agenda política durante la década de 1990 y finalmente se reformó en 1999 mediante la "Ley de las Pensiones y el Seguro de Invalidez" que incluía una serie de bonificaciones y sanciones para estimular la jubilación tardía. Los ejercicios de simulación, sin embargo, mostraron que la reforma de 1999 era insostenible a medio-largo plazo.

Al final del año 2006 la Comisión recomendó que Eslovenia lanzara una nueva reforma incluyendo incentivos adicionales para retrasar la edad de jubilación. Tal reforma estaba preparada, pero luego se vio retrasada y reducida de manera significativa.

Las razones para este decepcionante retraso fueron tres: (1) la opinión pública no estaba preocupada con la insostenibilidad a largo plazo, (2) hay una "parte pensionista" poderosa en el gobierno de coalición y, por último y no por ello menos importante, (3) la coordinación inter-ministerial no funcionó correctamente en esta ocasión. Estos factores ralentizaron el proceso de reforma a pesar de las opiniones de los encuestados de que el DICE es "una herramienta útil... que les proporciona a los responsables políticos el ímpetu necesario para llevar a cabo las reformas", y que la presión para la reforma se ve reforzada por el, más vinculante, Pacto de Estabilidad y Crecimiento.

68 Fuente: Equipo de evaluación. 
También se explica otro mecanismo a continuación bajo la denominación de “conformar o enmarcar los temas y cuestiones de las políticas". Este punto estaba oculto o implícito al comienzo del análisis aunque desarrolla un papel importante.

\subsubsection{Presión inter-pares}

Pagani (2002: 5-6) enumera tres elementos que pueden crear presión en los gobiernos: "(1) una combinación de recomendaciones formales y el diálogo informal entre países pares; (2) el escrutinio público, las comparaciones y, en algunos casos, incluso el ranking de países”; y “(3) el impacto de todo lo anterior sobre la opinión pública nacional, las administraciones nacionales y los responsables políticos”.

En este informe, denominamos a este primer punto: presión inter-pares. Este concepto abarca el diálogo estructurado entre los países pares que desembocan en recomendaciones. La presión puede ser ejercida sobre los responsables políticos involucrados, durante las reuniones de coordinación y se puede transmitir a los demás responsables políticos en el país.

Al segundo punto lo hemos llamado: presión pública. Dicho concepto engloba el escrutinio público, las tablas de rankings y la referencia a las recomendaciones en los debates públicos. En este caso, los gobiernos están expuestos a la presión de la opinión pública nacional. Este segundo concepto se abordará en el siguiente apartado.

La presión de grupo puede ser ejercida sobre los responsables políticos que asisten a las reuniones de coordinación que luego son transmitidas a los demás responsables políticos de sus respectivos países.

Varios de los estudios revisados tienden a minimizar el papel de la presión interpares, por ejemplo,

- “Los Comités: como el Comité de Política Económica y el Comité de Empleo pueden jugar un papel [de presión de grupo], pero los debates con los miembros del Comité... sugieren que solo disponen de un tiempo limitado para dedicarlo a los Programas Nacionales de Reforma (PNR) y su posterior seguimiento. Por otra parte, los contactos bilaterales de la Comisión con los Estados miembros parecen haber sido bastante productivos. En general, la impresión obtenida es que la revisión inter pares es un buen principio pero es difícil llevarla a cabo con la suficiente profundidad como para ser útil" (Parlamento Europeo, 2006, p. 58).

- “El Método Abierto de Coordinación parece facilitar el desarrollo de culturas educativas entre los Estados miembros, la Comisión y los agentes sub-nacionales y trans-nacionales. Tales culturas educativas enmarcan el compartir la información, la resolución de problemas, objetivos comunes, acciones conjuntas, compromisos mutuos y responsabilidad mutua; utilizando una combinación de 
indicadores, estandares, objetivos, planes nacionales, aprendizaje de pares y revisión inter pares. El "señalar con el dedo o dar fama" mediante mecanismos de evaluación comparativa que se practica hoy en día tiende a reforzar el juego y el posicionamiento de la oposición ya que tiene que fomentar un comportamiento deseable" (citado en la Comisión Europea, 2005).

La encuesta nos hizo comprender cómo las recomendaciones de la Comisión pueden crear cierto tipo de presión de grupo. Cerca del 60\% de los encuestados reconocen que la recomendación tiene cierta influencia, pero es difícil delimitar cómo ocurre dicha influencia.

Los cuatro casos estudiados (dieciséis estudios de países) ofrecen nuevas oportunidades para entender si las recomendaciones funcionan (véase la Tabla 3). En la mayoría de los casos, la presión de grupo no ejerció ningún papel en la aprobación de las reformas o no contribuyó a su desbloqueo. El caso de una mejor regulación en los Países Bajos es una excepción en el sentido de que las recomendaciones fueron usadas por los responsables políticos para vencer la resistencia en la administración pública. Sin embargo, este tipo de presión es muy diferente de la que se supone que se aplica.

\section{TABLA 3}

Presión del grupo: demostrando la hipótesis'

\begin{tabular}{|c|c|c|c|c|}
\hline Envejecimiento & $\begin{array}{c}\text { Mejor } \\
\text { regulación }\end{array}$ & $\begin{array}{c}\text { Profesiones } \\
\text { Reguladas }\end{array}$ & $\begin{array}{c}\text { Aprendizaje } \\
\text { permanente }\end{array}$ & $\begin{array}{c}\text { Hipótesis } \\
\text { confirmada }\end{array}$ \\
\hline DE & UK & GR & DK & Sí \\
\hline OS & NL & IT & EE & Más bien sí \\
\hline SL & DE & IE & NL & Más bien no \\
\hline PT & PL & FR & GR & No \\
\hline \multicolumn{4}{r|}{} \\
\cline { 4 - 5 }
\end{tabular}

Fuente: Equipo de evaluación.

\subsubsection{La presión pública}

Otra clase de presión puede ser ejercida por la opinión pública si las reformas, sus progresos y las recomendaciones se debaten ampliamente en los foros públicos. Una discusión tan abultada puede ser eficaz para incrementar la conciencia social de la necesidad de dichas reformas y aumentar la apropiación de las mismas. Estas hipótesis se examinan en los siguientes párrafos.

12 La hipótesis bajo examen es la siguiente: el debate del borrador de recomendaciones induce a los responsables políticos involucrados a reconocer que algunas reformas están bloqueadas y les lleva a desarrollar esfuerzos en desbloquearlos. Un gran número de responsables políticos preocupados aúnan sus esfuerzos en desbloquear las reformas, que finalmente son desbloqueadas, aprobadas e implementadas (véanse el Apartado 2.3 y la Figura 1). 
Primero, el análisis nos muestra que los Estados miembros generalmente han jugado a involucrar a los agentes sociales, parlamentos nacionales y autoridades regionales en el diseño y el seguimiento de los programas de reformas. Las recomendaciones generalmente se difunden y comentan en los medios de comunicación. No obstante, la visibilidad general del instrumento de "coordinación suave” para el público general es muy baja.

La apertura del proceso de "coordinación suave" fue reconocido por el Parlamento Europeo que "agradece la participación del parlamento y los agentes sociales", pero "lamenta la visibilidad, aún pobre" del proceso de coordinación (parlamento Europeo, 2007b). La falta de visibilidad se comenta aún más en un estudio Parlamentario en estos términos: "Si el público en general lo ignora [las estrategias relativas a Lisboa] (y la realidad es que éste es el caso), entonces este mismo público no podrá articular demandas de mejora a los gobiernos" (Parlamento Europeo, 2007c: 32).

El hecho de que el proceso de coordinación es relativamente abierto ¿implica que las reformas sean facilitadas y/o desbloqueadas? La encuesta no ayuda a aclarar este punto. Sólo tres de los entrevistados en tres países diferentes mencionan que una apropiación aumentada y la presión pública han ayudado al proceso de reforma. Por ejemplo: “El proceso DICE es positivo para nuestra organización porque podemos basar nuestra posición en las críticas de la Comisión y en la comparación con otros países"; "las recomendaciones se revisan en la prensa nacional y seguidamente se usan como argumento por o en contra de las medidas implementadas por el gobierno".

Del mismo modo, los cuatro casos de estudio y los dieciséis países de estudio no muestran claramente si se confirman o no las hipótesis (véase la Tabla 4).

TABLA 4

Apropiación ampliada y presión social: demostrando la hipótesis'13

\begin{tabular}{|c|c|c|c|c|}
\hline Envejecimiento & $\begin{array}{c}\text { Mejor } \\
\text { regulación }\end{array}$ & $\begin{array}{c}\text { Profesiones } \\
\text { Reguladas }\end{array}$ & $\begin{array}{c}\text { Aprendizaje } \\
\text { permanente }\end{array}$ & $\begin{array}{c}\text { Hipótesis } \\
\text { confirmada }\end{array}$ \\
\hline DE & UK & GR & DK & Sí \\
\hline OS & NL & IT & EE & Más bien sí \\
\hline SL & DE & IE & NL & Más bien no \\
\hline PT & PL & FR & GR & No \\
\hline \multicolumn{2}{|r|}{}
\end{tabular}

Fuente: Equipo de evaluación.

13 La hipótesis bajo examen es la siguiente: un gran número de interesados y grupos de interés debaten los programas de reforma y las recomendaciones. Desarrollan esfuerzos convergentes para fomentar las reformas, desbloqueándolas cuando es necesario. Estos esfuerzos tienen éxito sobre los responsables políticos preocupados desbloqueando las reformas, que finalmente son desbloqueadas, aprobadas e implementadas (véanse el Apartado 2.3 y la Figura 1). 
El análisis, por tanto, ha sido más profundo gracias a un estudio más de cerca de los estudios de países más significativos. En general, la evaluación no muestra ninguna instancia en la que el instrumento de "coordinación suave" no hubiera contribuido a desbloquear una reforma. La razón es que la presión ejercida no tiene una magnitud del mismo orden que los factores poderosos que dirigen las políticas internas en los Estados miembros.

Este punto no puede considerarse como un resultado robusto ya que el equipo de evaluación no se ha provisto de las herramientas de investigación que le habrían permitido analizar en profundidad las interacciones entre las recomendaciones y las políticas nacionales.

Por último, se entiende que las recomendaciones no ejercen presión en los gobiernos sino que, más bien, refuerzan la legitimidad de los promotores de la reforma, que es una pequeña diferencia en el conjunto de fuerzas que impulsan las políticas nacionales. Cuando se abre una ventana política a la reforma, entonces esta pequeña fuerza contribuye al proceso de aceleración, pero no es lo suficientemente fuerte como para abrir una ventana cerrada.

Esta interpretación es coherente con todas las pruebas disponibles derivadas de documentos de segunda mano, entrevistas y estudios de caso.

\subsubsection{La conformación de los temas y cuestiones de las políticas}

La conformación de un tema de una política pública implica que se alcance un amplio (y muchas veces informal) consenso en materia de:

- Los conceptos por los que los desafíos y problemas son identificados y debatidos;

- Los objetivos e indicadores por los que se establecen los objetivos y se hacen las comparativas;

- El modelo lógico ${ }^{14}$ explicando por qué unas soluciones funcionan y otras no;

- Las buenas prácticas.

Para la elaboración de políticas, el asunto relacionado con las necesidades de determinadas políticas tiene que estar debidamente conformado o enmarcado ${ }^{15}$. Las

14 Un modelo lógico es una serie de cadenas causa-efecto que conectan a un determinado tipo de reformas con su(s) resultado(s) pretendido(s). El término nada tiene que ver con el modelado econométrico, una aproximación que pretende proporcionar estimaciones cuantitativas de los resultados probables o reales, y que se utiliza cada vez más en los análisis relativos a Lisboa.

15 El término "frame" se ha tomado prestado del Schön y Rein (1994). Los autores defienden que el enmarcado o configuración es necesaria para hacer inteligible una situación problemática. Los marcos habitualmente expresan un diagnóstico y una solución de tal forma que la hace parecer una obviedad. "Este sentido de la obviedad de lo que está mal y lo que debe ser arreglado es el distintivo de los marcos políticos". 
reformas son considerablemente facilitadas cuando el asunto político está enmarcado de forma consensuada.

La cuestión de si las directrices son lo suficientemente operativas puede ser comprendido de la siguiente forma: "¿Se identifican las directrices individuales con marcos suficientemente desarrollados?" Está claro que la respuesta sería: "no todos". También está claro que la mejora de las directrices en este aspecto no es solo una cuestión de escritura. Más bien al contrario, este análisis muestra que hacen falta diez años o más para que un asunto de las políticas (policy issue) se enmarque de una manera lo suficientemente consensuada y práctica para poder elaborar políticas.

El análisis de la conformación de consenso en los estudios de casos muestran que los marcos pueden originarse en esferas muy diferentes (véase el Cuadro 5). En el caso del envejecimiento, el proceso se ha desarrollado a escala comunitaria en el marco de un proceso de "coordinación suave", es decir, principalmente en los grupos de trabajo de los comités. En contraste, el enmarcado de la cuestión de "legislar mejor” se ha dado en una esfera diferente a nivel europeo, pero no comunitario.

\section{CUADRO 5}

Conformando los temas y cuestiones de las políticas públicas que deben coordinarse

Los cuatro estudios de casos incluyeron entrevistas con una serie de agentes de trece países y fue sorprendente oír que se repetían las ideas como: “todas las partes creen en...”, “... es un tipo de paradigma”, “... es una visión compartida a la que nunca nos referimos”, “... se forma mediante un consenso internacional”, “... ayudó a formalizar nuestra reflexión y trabajo”, “... ayuda a los responsables políticos a pensar de una manera diferente”.

En el caso del envejecimiento, el grupo de trabajo del Comité de Política Económica empezó a enmarcar los conceptos, métodos e indicadores que permitían que los Estados miembros evaluaran sus retos. Entonces la Presidencia alemana inició otro grupo de trabajo con la intención de llegar a un consenso en las soluciones que funcionan. El proceso de conformación comenzó en 1999 y ahora está cerca de su final.

En el caso de una mejor regulación, el problema primero fue enmarcado por los Países Bajos y los países escandinavos. Estos precursores introdujeron los conceptos, los métodos y los indicadores a través de los cuales se debate la agenda de reformas en toda Europa. Entonces el Reino Unido realizó unos esfuerzos intensos en revisar y desarrollar más las soluciones que funcionan. Por todas partes, el consenso se buscó mediante una serie de redes internacionales. El proceso de enmarcado comenzó en 2000 y el desarrollo en el ámbito Comunitario llevará unos años más. Además, el caso de estudio muestra que aún no hay un consenso acerca de las soluciones que funcionan.

Un caso de aprendizaje permanente está más cerca del caso del envejecimiento en el sentido de que el asunto fue enmarcado en el ámbito Comunitario, principalmente por la Comisión, que desarrolló el indicador clave. El proceso comenzó en 1995, pero un número de encuestados consideran que aún no está cerca de su final.

Fuente: Equipo de evaluación. 
Lo que debemos considerar ahora es el papel de un tercer ámbito, las instituciones multilaterales como la OCDE, el FMI, el Banco Mundial, la OIT, etc. Los cuatros estudios de caso demuestran que estas instituciones tienen su propia influencia, a veces a través de instrumentos paralelos de "coordinación suave". Las redes de investigación internacionales y comunidades de conocimiento ofrecen otro lugar para enmarcar el consenso que es visible en el caso de una mejor regulación (Cuadro 6).

\section{CUADRO 6}

\section{Homenaje a las instituciones multilaterales}

El asunto del envejecimiento ya estuvo sujeto a cierto tipo de "coordinación suave" bajo los auspicios del FMI al principio de la década de 1990, es decir, casi diez años antes de que se afrontara a nivel Comunitario en 1999. A lo largo de los últimos ocho años, la OCDE también ha conducido un trabajo paralelo y bien coordinado sobre el envejecimiento, lo cual, evidentemente, ayudó a enmarcar el asunto, aunque se considera que el proceso a nivel Comunitario ha hecho la mayor contribución.

La apertura de las profesiones reguladas a la competencia es un asunto que se ha tratado tanto al nivel de la OCDE como Comunitario. Mientras las instituciones Comunitarias aplican una combinación de instrumentos duros (legislación) y suaves (MAC), la OCDE se apoya exclusivamente en la coordinación suave. La influencia de la OCDE es patente en Irlanda donde se dice que la mejora fue inducida por una recomendación de la OCDE.

El asunto del aprendizaje permanente actualmente se está debatiendo a nivel Comunitario, pero Europa no es el único ámbito en el que se enmarca este asunto. Uno de los objetivos estratégicos de la Dirección de Educación de la OCDE es fomentar el aprendizaje permanente y la mejora de sus vínculos con la sociedad y la economía. La Organización Internacional del Trabajo también trabaja activamente en este ámbito.

El asunto de una mejor regulación ha sido enmarcado por un grupo de países europeos precursores. No obstante, debemos añadir que la OCDE allanó el camino al emitir un análisis acerca de los diversos regímenes legislativos de los países miembros al comienzo de la década de 1990. Las "Recomendaciones para Mejorar la Calidad de la Regulación Gubernamental" se publicaron en 1995 y se han actualizado constantemente desde entonces. SIGMA (Support for Improvement in Governance and Management - Apoyo para la Mejora de la Gobernabilidad y la Gestión), una iniciativa conjunta de la OCDE y la UE, ya en 1992, propuso una mejor regulación en los nuevos Estados miembros. Por último, el proyecto "Doing Business" del Banco Mundial merece ser mencionado dado que dio forma a los conceptos, indicadores y rankings que actualmente se usan por todo el mundo.

Fuente: Equipo de evaluación.

\subsubsection{Segunda conclusión: El instrumento de "coordinación suave" no funciona como se pretendía}

Como se muestra en las figuras infra, el instrumento no funciona precisamente como esperaban los entrevistados en la fase inicial de la evaluación (véase la Figura 1).

En primer lugar, la evaluación revela un mecanismo llamado "Conformación de 74 las cuestiones políticas", que rara vez es citado por los interesados y los documentos, 
pero que juega un papel fundamental en la eficacia de la coordinación. Sin embargo, enmarcar un asunto político no es sencillo dado que habitualmente enmarcar una política pública para que sea ampliamente aceptada de una forma lo suficientemente práctica para realizar políticas suele llevar diez años. En la mayoría de los casos, este proceso implica varios ámbitos de debate y coordinación, es decir, no sólo la Comunidad y su proceso de coordinación de Lisboa, sino también instituciones multilaterales, comunidades académicas de conocimiento y incluso, en algunos casos, un grupo ad hoc de países precursores europeos.

En segundo lugar, la evaluación arroja dudas acerca de la eficacia de los mecanismos de "presión" que se citan constantemente como un mecanismo fundamental. Se descubre que (1) la presión interpares no funciona, y (2) la presión pública tampoco parece funcionar, al menos en el sentido de contribuir a desbloquear las reformas políticas. La razón es que la presión ejercida no tiene una magnitud del mismo orden que los factores poderosos que dirigen las políticas internas en los Estados miembros. El segundo resultado relacionado con la presión pública no es tan robusto debido a una limitación en el método de evaluación.

\section{CONCLUSIONES}

\subsection{Instrumento suave, efectos blandos}

Este apartado resume los apartados 3.1.4 y 3.2.4 mencionados anteriormente ${ }^{16} \mathrm{y}$ establece las conclusiones acerca de la eficacia del instrumento.

La evaluación confirma que la "coordinación suave" es eficaz para dar forma a la agenda de reformas y para fomentar el aprendizaje mutuo. A la vista de la información recogida y el análisis anterior, se puede decir que el instrumento tiene un impacto marginal sobre las agendas de reformas en la mayoría de los Estados miembros ${ }^{17}$. No obstante, hay una serie de otros factores que hacen una mayor contribución al aprendizaje mutuo e incluso más a la conformación de las agendas políticas.

La evaluación revela un mecanismo denominado "conformando los temas de las políticas", que juega un papel fundamental en la eficacia de la coordinación. En la mayoría de los casos, este proceso implica varios ámbitos de debate y coordinación, es decir, no solo la Unión Europea y su proceso de coordinación, sino también instituciones multilaterales, comunidades académicas de conocimiento e incluso, en algunos casos, un grupo ad hoc de países precursores europeos.

16 En palabras del análisis de contribución, esta es la "historia de contribución".

17 Esta valoración no está en línea con la de I.Begg en su informe: "Sin embargo, si es para proporcionar valor añadido a lo que las políticas nacionales obtendrían en su ausencia, el MAC también debe englobar la forma de cambiar lo que harían los Estados Miembros en cualquier caso y no es, de ninguna forma, evidente que lo logre el marco de Lisboa II” (Parlamento Europeo, 2006: 56). 
Por último, la evaluación arroja dudas acerca de la eficacia de los mecanismos de “presión”. Se demuestra que (1) la presión inter pares no funciona, y (2) la eficacia de la presión pública es cuestionable, al menos en el sentido de contribuir a desbloquear las reformas políticas.

La razón es que la presión ejercida no tiene una magnitud del mismo orden que los factores poderosos que dirigen las políticas internas en los Estados miembros.

En general, la "coordinación suave” es eficaz, pero no más de lo que cabría esperar de un instrumento tan blando, y no funciona como se esperaba.

Las mismas conclusiones se expresan gráficamente en la Figura 2. Dicha Figura muestra la interpretación del equipo de evaluación de qué funciona y qué no. En línea con la lógica del paso-a-paso del análisis de contribución, los resultados siguen las hipótesis lógicas que se obtuvieron al principio de la evaluación (véase la Figura 1).

\section{FIGURA 2}

\section{Lo que funciona y lo que no}

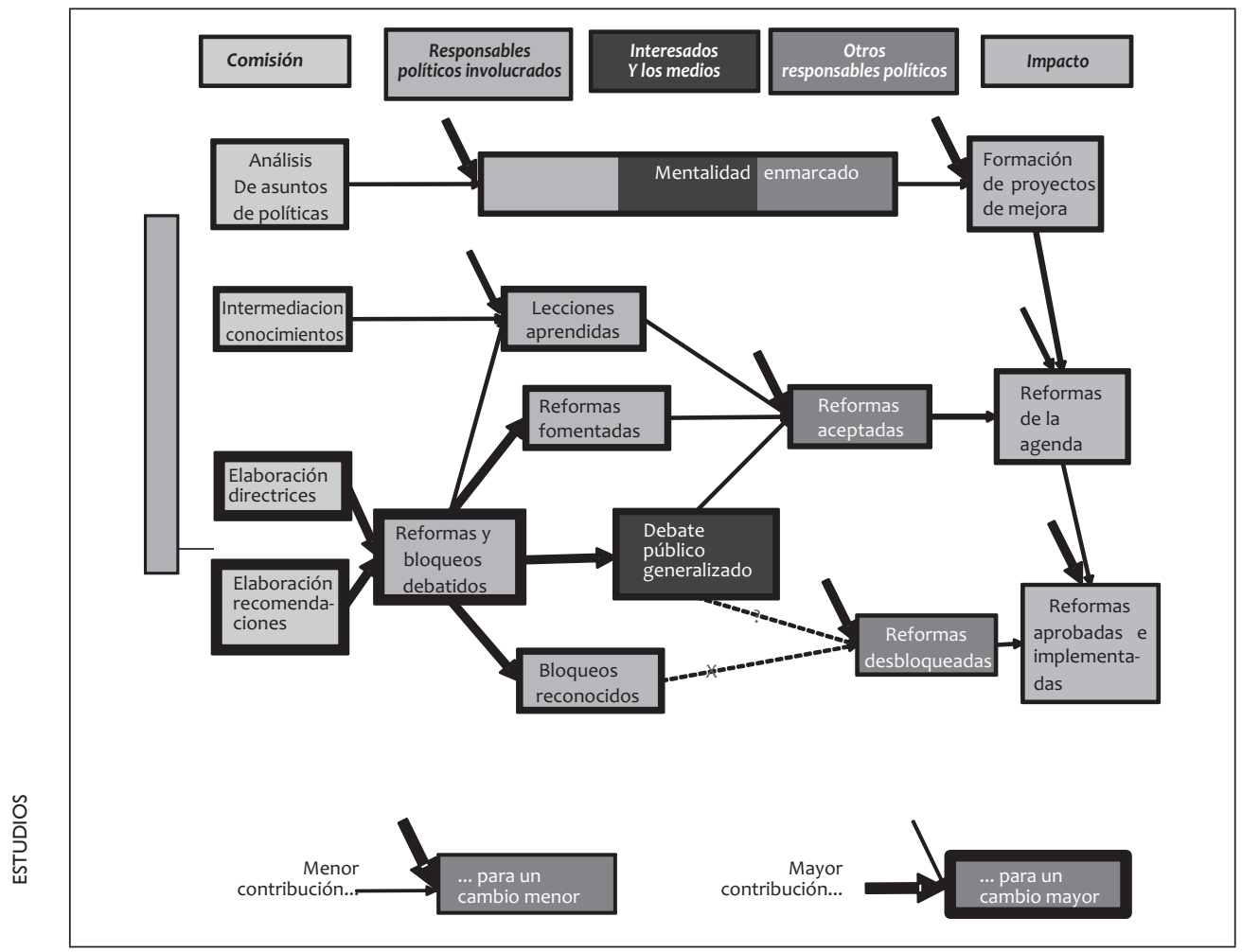

Fuente: Equipo de evaluación. La interpretación del equipo de evaluación acerca de la relativa importancia de los factores de contribución (flechas gruesas y finas) para observar los cambios y eventos. Dos hipótesis causa-efecto (flechas de putos) son falseadas $(\mathrm{X})$ o cuestionadas. 


\subsection{El debate democrático: una cuestión pendiente}

Schäffer (2006) afirma que la "coordinación suave" "es la primera y primordial forma de fomentar los compromisos en ausencia de acuerdos sustanciales... Las organizaciones internacionales se han apoyado repetidas veces en "legislación suave" (soft law) para superar las diferencias entre sus miembros. El FMI, la OCDE y la EU introdujeron la "coordinación suave" en momentos de crisis institucional para evitar una ruptura de las negociaciones".

Este punto de vista es sorprendente si se conecta a una lección clave aprendida durante esta evaluación, es decir, que una visión compartida (marco) es un canal principal para el funcionamiento de la "coordinación suave".

Parece haber una contradicción en el hecho de que la conformación sea el motor de un mecanismo que se usa en caso de desacuerdos políticos. Esta contradicción probablemente se pueda resolver si se replantea de la siguiente forma: Si un Estado miembro necesita abordar un asunto político en común y no puede acordar si hacerlo en un marco regulatorio formal, entonces la "coordinación suave" es una alternativa. La "coordinación suave" requiere una visión compartida de los asuntos políticos en cuestión pero su visión común puede seguir siendo informal en gran medida.

Teniendo en cuenta que los marcos "suelen transmitir un diagnóstico y una solución de tal forma que puedan parecer evidente” (véase la nota al pie número 15), cabe suponer que la "coordinación suave" es eficaz en tanto en cuanto esté protegida de las controversias políticas.

Esta idea también está recogida en un documento Parlamentario en los siguientes términos: "Es innegable que la Estrategia de Lisboa se basa en una visión particular de cómo debería evolucionar la economía de la UE. Uno de los misterios de Lisboa es que esta visión tiende a permanecer, en gran medida, sin oposición y con pocas intenciones de proponer unas visiones alternativas o de condenarlo" (Parlamento Europeo, 2006: 63).

El funcionamiento de la "coordinación suave" puede resultar paradójica a la luz de las afirmaciones del Parlamento Europeo al solicitar una mayor participación en el proceso de "coordinación suave" (Parlamento Europeo 2007b: 21). ¿Hasta qué punto puede la "coordinación suave" estar sujeta a un control democrático, dado que los debates políticos tienen el propósito de retar públicamente el consenso y que este consenso es lo que hace que funcione la "coordinación suave"?

\section{REFERENCIAS BIBLIOGRÁFICAS Y DOCUMENTALES}

Comisión Europea (2005), El Análisis del Impacto de la Evaluación Comparativa y las Acciones de la UE en el Método Abierto de Coordinación. Bruselas: Comisión Europea. 
Comisión Europea (2006), Informe de Innovación Europeo 2006. Bruselas: Comisión Europea. En línea: <www.trendchart.org> (Consulta: septiembre 2007).

Hodson, D. y Maher, I. (2001), "The Open Method as a New Mode of Governance: The Case of Soft Economic Policy Co-ordination”, Journal of Common Market Studies, 39(4): 719-746.

Mayne, J. (1999), “Addressing Attribution through Contribution Analysis: Using Performance Measures Sensibly", Canadian Journal of Program Evaluation, 16(1): 1-24.

Mayne, J. (en prensa), "Contribution Analysis: Addressing Cause and Effect in Simple and Complex Settings", eds., R. Schwartz, K. Forss, y M. Marra en Evaluating the Complex. New Brunswick: Transaction Publishers.

OCDE (2007), El criterio de evaluación de la OCDE del mecanismo de evaluación interna. París: OCDE.

Parlamento Europeo / ECON (2006a), La Implementación de las Directrices Integradas durante el Ciclo Político 2005/2006 (Informe elaborado por I. Begg). Bruselas: Parlamento Europeo.

Parlamento Europeo / ECON (2006b), La Implementación de las Directrices Integradas durante el Ciclo Político 2006/2007 (Informe elaborado por I. Begg). Bruselas: Parlamento Europeo.

Parlamento Europeo / CEMA (2007a), Resolución sobre la Situación de la Economía Europea, Informe preparatorio sobre las directrices políticas generales para 2007. Bruselas: Parlamento Europeo.

Parlamento Europeo / CEMA (2007b), Resolución sobre la Situación de la Economía Europea. Informe provisional sobre las directrices políticas generales para 2008. Bruselas: Parlamento Europeo.

Pisani-Ferry, J. y Sapir, A. (2006), Last exit to Lisbon. Informe Bruegel preparado a instancias de la Presidencia Austriaca. Bruselas: Bruegel.

Pagani, F. (2002), Peer review: A tool for co-operation and change. An Analysis of an OECD working method. París: OCDE.

Schäffer, A. (2006), "Resolving Deadlock: Why International Organisations Introduce Soft Law”, European Law Journal, 12(2): 194-208.

Schön, D.A. y Rein, M. (1994), Frame Reflection: Towards the Resolution of Intractable Policy Controversies. Nueva York: Basic Books.

Recibido: 18 de febrero de 2010

Aceptado: 22 de marzo de 2010 